\title{
Effectiveness of extracorporeal shockwave therapy in three major tendon diseases
}

\author{
Christian Carulli $^{1} \cdot$ Filippo Tonelli $^{1} \cdot$ Matteo Innocenti $^{1} \cdot$ Bonaventura Gambardella $^{1}$ \\ Francesco Muncibi ${ }^{1} \cdot$ Massimo Innocenti ${ }^{1}$
}

Received: 15 January 2015/ Accepted: 11 June 2015/Published online: 2 July 2015

(c) The Author(s) 2015. This article is published with open access at Springerlink.com

\begin{abstract}
Background Extracorporeal shockwave therapy is a conservative treatment for several painful musculoskeletal disorders. The aim of the study was the assessment of the relief from pain by the shockwave therapy in a population of consecutive patients affected by specific pathologies.

Materials and methods A group of consecutive patients were studied and treated. They were affected by calcific tendonitis of the shoulder (129 patients), chronic Achilles tendinopathy (102 patients), and lateral epicondylitis of the elbow (80 subjects). Each patient had 3 applications with a monthly interval, and was followed up at 1, 6, and 12 months after treatment. Results were evaluated by the numeric rating scale (NRS) in all cases, the Constant Murley Score for the assessment of the shoulder function, the American Orthopaedic Foot and Ankle Society Score for subjects affected by chronic Achilles tendinopathy, and the Oxford Elbow Score for those affected by a lateral epicondylitis of the elbow.

Results One year after treatment, the results were considered satisfactory with an almost complete resolution of symptoms. There were statistically significant results at the 12-month follow-ups regarding the mean NRS score (from 6.25 to 0.2), the Constant Murley Score (from 66.7 to 79.4), the Oxford Elbow Score (from 28 to 46), and the AOFAS (from 71 to 86).

Conclusions Extracorporeal shockwave therapy may be considered a safe, economic, and effective treatment for
\end{abstract}

Christian Carulli

christian.carulli@unifi.it

1 Orthopaedic Clinic, University of Florence, Largo P. Palagi 1, 50139 Florence, Italy several chronic musculoskeletal disorders, allowing satisfactory pain relief and improvement of function ability. Level of evidence Level IV.

Keywords Extracorporeal shockwave $\cdot$ Calcific tendonitis of the shoulder - Achilles tendinopathy . Epicondylitis

\section{Introduction}

Extracorporeal shock wave therapy (ESWT) is one of the great advances in orthopaedics over the last 20 years [1]. Initially indicated for the treatment of kidney stones [2], it has been applied in cases of bone non-unions, and then in several musculoskeletal disorders, given the satisfactory clinical outcomes reported in different randomized clinical trials and cohort studies. The main indications have been the following: lateral epicondylitis of the elbow, calcific tendonitis of the rotator cuff, plantar fasciitis, Achilles and patellar tendinopathy, and pubalgia [1, 3-13]. A reduction of pain and a good recovery of articular function have been obtained in most cases [13-17], even if in high-level athletes a more aggressive strategy is recommended to allow a quick return to sports activities [18].

The mechanism by which ESWT may produce a clinical effect is still uncertain. Several theories have been proposed: a mechanical effect by increasing the pressure in the calcium deposition causing fragmentation; a molecular effect with induction of an inflammatory response with neovascularization and then a chemotactic action and phagocytosis of calcific deposits; an analgesic effect by inhibiting the activation of the serotonergic system, and peripheral denervation. Probably, a combination of angiogenic and analgesic effects explains the overall outcomes 
on the target tissues [7, 8, 19-23]. Direct and indirect biologic effects of ESWT vary proportionally to the amount of energy and to the type of frequency applied; moreover, the shockwave driving tool influences the induced modifications on the target tissue [24].

The aim of the present retrospective study is the evaluation of the clinical outcomes in a population of patients affected by common musculoskeletal disorders treated by ESWT.

\section{Materials and methods}

From January 2011 to March 2013, 311 consecutive patients were selected and treated by ESWT for specific painful musculoskeletal disorders at the authors' institution. One-hundred and twenty-nine were affected by a calcific tendonitis of the shoulder, 102 by an Achilles tendinopathy, and 80 by a later epicondylitis of the elbow.

The mean age was 48.5 (range 19-80); 230 were male, and 81 female. Inclusion criteria were: adult patients with clinical and instrumental diagnosis of lateral epicondilytis of the elbow, chronic Achilles tendinopathy, and calcific tendonitis of the shoulder; persistent symptoms for at least 3 months; failure or partial resolution of symptoms after conservative (medical and physical) treatment; no recent history of trauma or chronic joint instability; no recent related surgery.

Exclusion criteria were: patients with a clinical but not instrumental diagnosis of any tendon disease; subjects who had not tried any conservative approach; subjects referring an inadequate duration of proper medical or physical treatments. The institutional review board allowed the retrospective analysis of patients' data and outcomes. Demographic data of the selected patients are reported in Table 1.
Pain assessment in all patients was conducted before treatment by an 11-point numeric scale (numeric rating scale, NRS). The clinical evaluation was conducted by the Constant Score for the assessment of shoulder function [25]; the American Orthopaedic Foot and Ankle Society Score (AOFAS) [26] for subjects affected by chronic Achilles tendinopathy; and the Oxford Elbow Score [27] for those suffering a lateral epicondylitis of the elbow. All patients gave their consent to the treatment and follow-up.

A single device generating shockwaves (ReflecTron ${ }^{\circledR}$, HMT, Switzerland) was used in all cases. The energy level and number of shots were adapted to the specific pathology according to the protocols supplied by the manufacturer. Each patient had 3 ESWT applications at monthly intervals. Each session consisted of 2400 shockwave applications with an intensity depending on the site and the pathology observed (Table 2). No local anaesthesia was given before the treatment. All patients were treated by two experienced orthopaedic surgeons.

All subjects were followed up at 1, 6, and 12 months after the last application. The clinical evaluation consisted of NRS and function evaluation by the administration of the above mentioned specific scores (Table 3). Particular attention has been focused on the use of analgesic drugs, reported complications after the ESWT applications, and the need for any further instrumental study.

The statistical analysis was performed by a sample size calculation based on a priori assumption of $p=0.05$. All data were tested for the normal distribution using the Kolmogorov-Smirnov test. The Student t-test was used to perform the analysis for the scores, testing each disease separately. For each parameter, three coupled samples were calculated (before treatment- 1 month, before treatment -6 months, before treatment -12 months) (Table 3).

Table 1 Demographics and characteristics of the patients

\begin{tabular}{llll}
\hline & $\begin{array}{l}\text { Shoulder calcific tendonitis } \\
(n=129)\end{array}$ & $\begin{array}{l}\text { Achilles tendinopathy } \\
(n=102)\end{array}$ & $\begin{array}{l}\text { Elbow lateral } \\
\text { epicondylitis }(n=80)\end{array}$ \\
\hline Male/female & $92 / 37$ & $46 / 56$ & $45 / 35$ \\
Mean age (range) & $47.5(19-70)$ & $48(22-80)$ & $50(20-76)$ \\
Mean duration of symptoms (weeks) & $4.3(3-7)$ & $6.7(2-9)$ & $3.9(2-6)$ \\
Dominant side affected & 72 & 64 & 56 \\
Previous treatments (number of subjects) & & & 19 \\
NSAIDs & 31 & 24 & 28 \\
Other analgesics & 52 & 41 & 12 \\
Physical therapy & 21 & 16 & \\
\hline
\end{tabular}


Table 2 Active level of ESWT

\begin{tabular}{lc}
\hline Disease & Pulses and energy of ESWT \\
\hline Calcific tendonitis of the shoulder & 2400 pulses at $>0.20 \mathrm{~mJ} / \mathrm{mm}^{2}$ \\
Achilles tendinopathy & $\begin{array}{c}2400 \text { pulses at } 0.08-0.33 \mathrm{~mJ} / \\
\mathrm{mm}^{2}\end{array}$ \\
Lateral epicondylitis of the elbow & 2400 pulses at $<0.12 \mathrm{~mJ} / \mathrm{mm}^{2}$ \\
\hline
\end{tabular}

\section{Results}

Two-hundred and eighty-three patients completed the follow-up period. Twenty-eight subjects were lost: none of them was lost due to conditions or complications related to the procedures.

No complications were recorded. In 42 cases, the patients reported the presence of cutaneous bruises after the applications. The overall mean NRS score was 6.25 (range 4-9) before the treatment. One month after the first application, the mean NRS score was 4.9 (range 3-9), 1.2 at 6 months (range $0-3$ ), and finally 0.2 at 12 months (range 0-2). Considering single pathologies, patients showed an improvement in any score: mean NRS, mean Constant Murley Score for shoulders, mean Oxford Elbow Score for elbows, and mean AOFAS Score for feet (Table 3). Over the months of follow-up we recorded a progressive maintenance of results (Figs. 1,2). The use of pain regulating drugs was reported by 34 patients $(12.0 \%)$, with peak utilisation on the first 3 days, once daily. In 12 cases $(4.2 \%)$, the pain did not show a significant decrease so an ultrasound or MRI examination was necessary to understand the causes of the persistency of symptoms.

\section{Discussion}

Shockwave therapy represents an innovative approach for the management of painful chronic musculoskeletal diseases, particularly in the case of failure of a previous conservative treatment. This treatment has to be considered safe, minimally invasive, versatile, and with low costs [28].

In the present study, as reported in the literature, after a latency of days to a few weeks after treatment, all patients reported a clinical benefit, with a significant decrease of pain, improvement in function, and a fair use of analgesics. Focusing attention on the specific pathologies, our outcomes are in line with the latest reports.

Lateral epicondylitis of the elbow was treated by ESWT in five recent RCTs, mostly of high quality [29-33]. In two of these, no significant differences were found up to 48 weeks after the treatment between ESWT and placebo [31, 32]. Spacca et al. [31] found significant differences between ESWT and placebo on pain (0.5 versus 6.5) and grip strength (46 versus 36) 12 weeks after the treatment. Pettrone et al. [29] found similar significant differences in pain at the 12-week follow-up. Collins et al. [33] found significant difference in pain during activity in favour of the ESWT group. There is conflicting evidence for the effectiveness of ESWT versus placebo in the short term and evidence of no difference in effect on the mid-term and long-term follow-up.

Several studies have confirmed the benefits of ESWT for the treatment of calcific tendonitis of the shoulder [24, 34, 35]. Particularly, it has been reported that high-energy ESWT $\left(E F D \geq 0.28 \mathrm{~mJ} / \mathrm{mm}^{2}\right)$ are more effective than low-energy doses $\left(\mathrm{EFD}<0.28 \mathrm{~mJ} / \mathrm{mm}^{2}\right.$ ) in the improvement of the shoulder function and pain resolution.

Table 3 Clinical and functional scores

\begin{tabular}{|c|c|c|c|c|}
\hline & Baseline & 1 month & 6 months & 12 months \\
\hline \multicolumn{5}{|l|}{ Numeric rating scale (NRS) } \\
\hline Calcific tendonitis of the shoulder ${ }^{\mathrm{a}}$ & $6.5 \pm 1.4(4-9)$ & $\begin{array}{l}5.9 \pm 1.3(3-9)^{*} \\
p=00.013\end{array}$ & $\begin{array}{l}1.2 \pm 0.8(0-3)^{*} \\
p<0.001\end{array}$ & $\begin{array}{l}0.2 \pm 0.4(0-1)^{*} \\
p<0.001\end{array}$ \\
\hline Achilles tendinopathy ${ }^{\mathrm{a}}$ & $6.9 \pm 1.2(5-9)$ & $\begin{array}{l}5.3 \pm 1.1(4-8)^{*} \\
p<0.001\end{array}$ & $\begin{array}{l}1.7 \pm 0.8(0-3)^{*} \\
p<0.001\end{array}$ & $\begin{array}{l}0.3 \pm 0.5(0-2)^{*} \\
p<0.001\end{array}$ \\
\hline Lateral epicondylitis of the elbow ${ }^{a}$ & $6.6 \pm 1.2(4-9)$ & $\begin{array}{l}4.2 \pm 1.0(3-6)^{*} \\
p<0.001\end{array}$ & $\begin{array}{l}0.9 \pm 0.8(0-3)^{*} \\
p<0.001\end{array}$ & $\begin{array}{l}0.1 \pm 0.3(0-1)^{*} \\
p<0.001\end{array}$ \\
\hline \multicolumn{5}{|l|}{ Functional scores } \\
\hline Constant Murley Score & $66.7 \pm 4.3(56-76)$ & $\begin{array}{l}73.7 \pm 3.9(59-78)^{*} \\
p=00.012\end{array}$ & $\begin{array}{l}78.3 \pm 2.6(64-80)^{*} \\
p<0.001\end{array}$ & $\begin{array}{l}79.4 \pm 1.4(70-80)^{*} \\
p<0.001\end{array}$ \\
\hline AOFAS & $71 \pm 5.6(63-80)$ & $\begin{array}{l}72 \pm 3.2(67-75)^{*} \\
p<0.001\end{array}$ & $\begin{array}{l}77 \pm 2.4(72-84)^{*} \\
p<0.001\end{array}$ & $\begin{array}{l}86 \pm 1.9(82-90)^{*} \\
p<0.001\end{array}$ \\
\hline Oxford Elbow Score & $28 \pm 2.7(23-35)$ & $\begin{array}{l}35 \pm 2.5(31-38)^{*} \\
p=0.0016\end{array}$ & $\begin{array}{l}42 \pm 2.6(36-47)^{*} \\
p<0.001\end{array}$ & $\begin{array}{l}46 \pm 2.6(42-50)^{*} \\
p<0.001\end{array}$ \\
\hline
\end{tabular}

* Paired Student t-test, compared to baseline $(p<0.05)$

${ }^{\text {a }}$ The use of pain regulating drugs was reported by 34 patients $(12.0 \%)$, with a peak of utilization in the first 3 days, once daily 
Fig. 1 NRS scores after treatment

Fig. 2 Constant Murley, AOFAS, and Oxford Elbow Scores after treatment
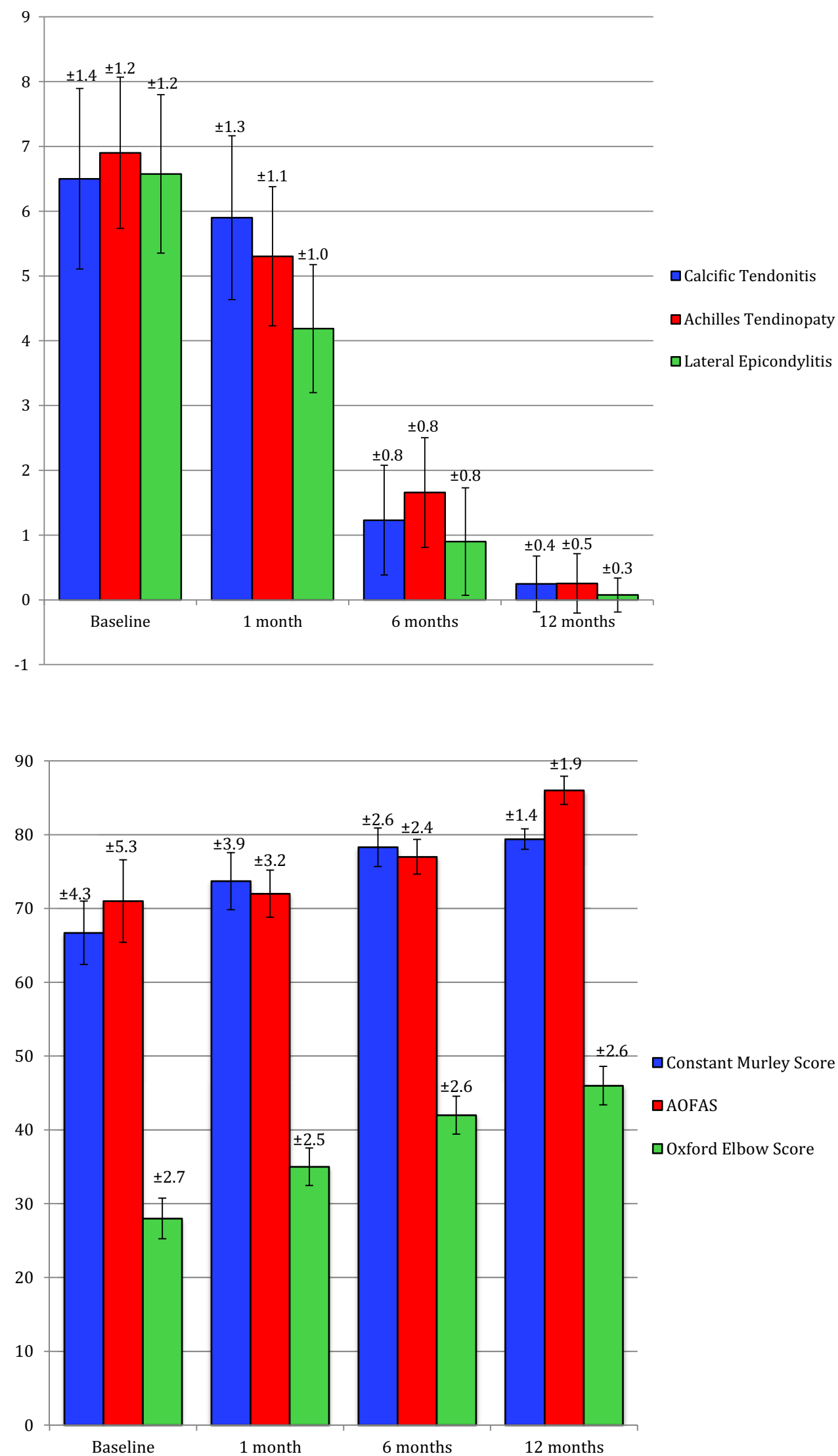
Gerdesmayer et al. [35] enrolled 144 patients with a randomized level of energy (high or low). Both types of ESWT resulted in a significant improvement at the 6-month evaluation, but high-energy ESWT induced a higher outcome on the Constant Murley Score. Calcific deposits disappeared in the same percentage of patients in both groups. Cacchio et al. [34] used a different score (University of California-Los Angeles UCLA Shoulder Rating Scale) to evaluate shoulder function after ESWT treatment versus placebo of calcific tendonitis of the shoulder. Significant differences in favour of ESWT versus placebo were reported at the 6-month follow-up.

ESWT is effective as a conservative approach in the treatment of chronic Achilles tendinopathy. This has been recently confirmed by some important RCTs [12, 36, 37]. Rasmussent et al. [12] showed improvements in the treatment with ESWT versus placebo at a 12-week follow-up. The mean AOFAS Score increased from 74 to 81 in the placebo group and from 70 to 88 in the ESWT group $(p=0.05)$. Better results were seen in the ESWT group at 8 and 12 weeks $(p=0.01$ and $p=0.04$, respectively). Rompe et al. [37] showed an improvement in the VISA-A score (specific for Achilles tendon pathologies) which increased in two groups: one with a treatment by eccentric loading exercises and one with eccentric loading + ESWT. The better outcomes were registered for the second group.

Despite the positive results, this study has some limitations. First of all, there was no patient randomization or use of placebo for any treatment. Moreover, there was no control group and the analysis of outcomes was performed without blind examiners. Our protocols were based on a 3-session ESWT application that, in our opinion, represents a reasonable approach, even if it is not the only approach. Finally, the diagnosis of each pathology was established by all authors, even if the ESWT applications were performed by two dedicated surgeons.

In conclusion, we believe that treatment with extracorporeal shockwaves may be a useful option in the management of several chronic musculoskeletal pathologies, particularly after the failure of a conventional approach. The wide spectrum of applications, the low related costs, and its safety represent the strength of this modern approach to the treatment of disabling musculoskeletal diseases.

\section{Compliance with ethical standards}

Conflict of interest The authors declare that they have no conflict of interest.

Ethical standards The study conforms to the 1964 Helsinki declaration and its later amendments; the study was approved by the institutional ethical review board; all the patients provided informed consent before being enrolled.
Open Access This article is distributed under the terms of the Creative Commons Attribution 4.0 International License (http://creativecommons.org/licenses/by/4.0/), which permits unrestricted use, distribution, and reproduction in any medium, provided you give appropriate credit to the original author(s) and the source, provide a link to the Creative Commons license, and indicate if changes were made.

\section{References}

1. Ogden JA, Alvarez RG, Levitt R et al (2001) Shock wave therapy (orthotripsy) in musculoskeletal disorders. Clin Orthop 387:22-40

2. Farsi HM, Mosli HA, Alzimaity M et al (1994) In situ extracorporeal shock wave lithotripsy for primary ureteric calculi. Urology 43:776-781

3. Rompe JD, Maffulli N (2007) Repetitive shock wave therapy for lateral elbow tendinopathy (tennis elbow): a systematic and qualitative analysis. Br Med Bulletin 83:355-378

4. Thomson CE, Crawford F, Murray GD (2005) The effectiveness of extra corporeal shock wave therapy for plantar heel pain: a systematic review and meta-analysis. BMC Musculoskelet Disord 6:19

5. Mouzopoulos G, Stamatakos M, Mouzopoulos D et al (2007) Extracorporeal shock wave treatment for shoulder calcific tendonitis: a systematic review. Skeletal Radiol 36:803-811

6. Roehrig GJ, Baumhauer J, DiGiovanni BF et al (2005) The role of extracorporeal shock wave on plantar fasciitis. Foot Ankle Clin 10:699-712

7. Ho C (2007) Extracorporeal shock wave treatment for chronic rotator cuff tendonitis (shoulder pain). Issues in Emerg Health Technol 96:1-4

8. Farr S, Sevelda F, Mader P et al (2001) Extracorporeal shockwave therapy in calcifying tendinitis of the shoulder. Knee Surg Sports Traumatol 19:2085-2089

9. Radwan Y, ElSobhi G, Badawy et al (2008) Resistant tennis elbow: shock-wave therapy versus percutaneous tenotomy. Int Orthop 32:671-677

10. Saxena A, Ramdath S, O'Halloran et al (2011) Extra-corporeal pulsed-activated therapy ("EPAT" sound wave) for Achilles tendinopathy: a prospective study. J Foot Ankle Surg 50:315-319

11. Rompe JD, Furia JP, Maffulli (2008) Mid-portion Achilles tendinopathy: current options for treatment. Disabil Rehabil 30:1666-1676

12. Rasmussen S, Christensen M, Mathiesen I et al (2008) Shockwave therapy for chronic Achilles tendinopathy: a double-blind, randomized clinical trial of efficacy. Acta Orthop 79:249-256

13. Rodriguez-Merchan EC (2013) The treatment of patellar tendinopathy. J Orthop Traumatol 14:77-81

14. Wang CJ, Ko JY, Chan YS et al (2007) Extracorporeal shockwave for chronic patellar tendinopathy. Am J Sports Med 35:972-978

15. Wang CJ, Wang FS, Yang KD et al (2006) Long term results of extracorporeal shockwave treatment for plantar fascitis. Am J Sports Med 34(4):592-596

16. Cacchio A, Rompe JD, Furia JP et al (2011) Shockwave therapy for the treatment of chronic proximal hamstring tendinopathy in professional athletes. Am J Sports Med 39:146-153

17. Daecke W, Kusnierczak D, Loew M (2002) Long-term effects of extracorporeal shockwave therapy in chronic calcific tendinitis of the shoulder. J Shoulder Elb Surg 11:476-480

18. Benazzo F, Marullo M, Zanon G et al (2013) Surgical management of chronic proximal hamstring tendinopathy in athletes: a 2 to 11 years of follow-up. J Orthop Traumatol 14:83-89

19. Wang CJ, Huang HY, Pai CH (2002) Shock wave enhances neovascularization at the tendon-bone junction. J Foot Ankle Surg 41:16-22 
20. Lian O, Dahl J, Ackermann PW et al (2006) Pronociceptive and antinociceptive neuromediators in patellar tendinopathy. Am $\mathbf{J}$ Sports Med 34:1801-1808

21. Ohtori $\mathrm{S}$ et al (2001) Shock wave application to rat skin induces degeneration and reinnervation of sensory nerve fibres. Neuroscience 315:57-60

22. Wang FS, Yang KD, Chen RF et al (2002) Extracorporeal shock wave promotes growth and differentiation of bone-marrow stromal cells towards osteoprogenitors associated with induction of TGF-beta1. J Bone Joint Surg Br 84:457-461

23. Chen YJ, Wurtz T, Wang CJ et al (2004) Recruitment of mesenchymal stem cells and expression of TGF-beta 1 and VEGF in the early stage of shock wave-promoted bone regeneration of segmental defect in rats. J Orthop Res 22:526-534

24. Ioppolo F, Tattoli M, Di Sante L et al (2012) Extracorporeal shock-wave therapy for supraspinatus calcifying tendinitis: a randomized clinical trial comparing two different energy levels. Phys Ther 92:1376-1385

25. Constant CR, Murley AH (1987) A clinical method of functional assessment of the shoulder. Clin Orthop Relat Res 214:160-164

26. Kitaoka HB, Alexander IJ, Adelaar RS et al (1994) Clinical rating systems for the ankle-hindfoot, midfoot, hallux, and lesser toes. Foot Ankle Int 15:349-353

27. Dawson J, Doll H, Boller I et al (2008) The development and validation of a patient-reported questionnaire to assess outcomes of elbow surgery. J Bone Joint Surg Br 90:466-473

28. Ioppolo F, Rompe JD, Furia JP et al (2014) Clinical application of shock wave therapy (SWT) in musculoskeletal disorders. Eur J Phys Rehabil Med 50:217-230
29. Pettrone F, McCall BR (2005) Extracorporeal shock wave therapy without local anesthesia for chronic lateral epicondylitis. J Bone Joint Surg Am 87:1297-1304

30. Staples MP, Forbes A, Ptasznik R et al (2008) A randomized controlled trial of extracorporeal shock wave therapy for lateral epicondylitis (tennis elbow). J Rheumat 35:2038-2046

31. Spacca G, Necozione S, Cacchio A (2005) Radial shock wave therapy for lateral epicondylitis: a prospective randomised controlled single-blind study. Eur Medicophys 41:17-25

32. Chung B, Wiley JP, Rose MS (2005) Long-term effectiveness of extracorporeal shockwave therapy in the treatment of previously untreated lateral epicondylitis. Clin J Sport Med 15:305-312

33. Collins EDH, Jafarnia KK (2011) A clinical study of extracorporeal shock waves (ESW) for treatment of chronic lateral epicondylitis. Curr Orthop Pract 22:185-192

34. Cacchio A, Paoloni M, Barile A et al (2006) Effectiveness of radial shock-wave therapy for calcific tendinitis of the shoulder: single-blind, randomized clinical study. Phys Ther 86:672-682

35. Gerdesmeyer L, Maier M, Loew M et al (2003) Extracorporeal shock wave therapy for the treatment of chronic calcifying tendonitis of the rotator cuff. JAMA 290:7-10

36. Lakshmanan P, O'Doherty DP (2004) Chronic achilles tendinopathy: treatment with extracorporeal shock waves. Foot Ankle Surg 10:125-130

37. Rompe JD, Furia J, Maffulli N (2009) Eccentric loading versus eccentric loading plus shock-wave treatment for midportion achilles tendinopathy: a randomized controlled trial. Am J Sports Med 37:463-470 